\title{
Application Research on Face Detection Technology based on OpenCV in Mobile Augmented Reality
}

\author{
Hu Peng \\ Oxbridge College, Kunming University of Science and Technology, \\ Kunming, china, 650106 \\ E-Mail:hpeng1025@sina.com
}

\begin{abstract}
Augmented Reality (AR) is a new computer application and human-machine interaction derived from virtual reality. It has been widely applied in social each domain. With the improvement on the performance and the quality of built-in camera of smartphones, it is possible to have the augmented reality system to come true. And with people entertainment needs growing, mobile augmented reality based on face detection has become a research hotspot. This paper discusses the development and typical technology of face detection technology and mobile augmented reality. A development framework of the face detection technique based on OpenCV in mobile augmented reality application is proposed. And this essay takes the AR face book show as an example analyzes the key technology in the process of development and proves its feasibility and effectiveness.
\end{abstract}

Keywords: OpenCV, face detection, Mobile augmented reality

\section{Introduction}

Face detection is a very challenging issue; the main difficulty is that the face is a kind of highly non-rigid target, such as appearance, facial expression, and color difference. There might be some people face appendages such as glasses, beard, etc. The face poses change evil, and there may be a shelter. Differences in nature of the image are to be detected, such as waiting for the resolution of the image, the quality of video recording equipment, etc.

\section{Face Detection Technology}

\subsection{Development}

Face detection find faces information in the video sequence or image and determine facial size, position, trajectory, attitude, and further human face eyes, lips and other features extraction process. The exploration of human face detection issue began in the late 1980s and early 1990s, mainly concentrated in still images. In recent years, the research mainly focused on the real-time face detection of the video stream. The Goal is trying quickly and accurately determines whether there was a face in the video image sequence under the complex background. If there is a face, it gives the facial information, dynamically tracks human faces. This is a kind of operation according to the common features of face samples mode for pattern recognition.

At present, under the common booster of the evolution of the man-machine interactive way, the development of biometric recognition and object detection research, face detection technology is more and more attention from academia and industry. Many countries have launched face detection research, mainly the United States, Europe, Japan, etc. Well-known Research institutions have the MIT Media 
lab, the AI lab, CMU Human-Computer Interface at Microsoft Research, the British Department of Engineering in University of Cambridge, etc. The main mechanism of domestic research on face detection with Tsinghua University, Harbin Industrial University, Beijing University of Technology, the Institute of Automation of the Chinese Academy of Sciences, and have achieved certain results. Thus it can be concluded that more and more attentions are paid to face detection technology all over the world.

\subsection{Typical Technology: OpenCV}

Open Source Computer Vision Library, OpenCV for short, is a cross-platform library computer vision based on open source distribution. It can run on Linux, Windows and Mac OS operating system. It's lightweight and efficient-makes up of a series of $\mathrm{C}$ function and a small amount of $\mathrm{C}++$ class, at the same time provides the Python, Ruby, MATLAB language interface; realize the many general algorithm of image processing and computer vision.

Very rich visual processing algorithm provided by the OpenCV, with $\mathrm{C}$ language to write and it part, combined with the characteristics of its open source, it does not need to add new external support, which can also complete the compilation of links to generate executable program. As a result, many people use it to do transplant of the algorithm. Whether still images and video sequence images, using OpenCV function and classifier are relatively easy to face detection, the process is shown in Figure 1.

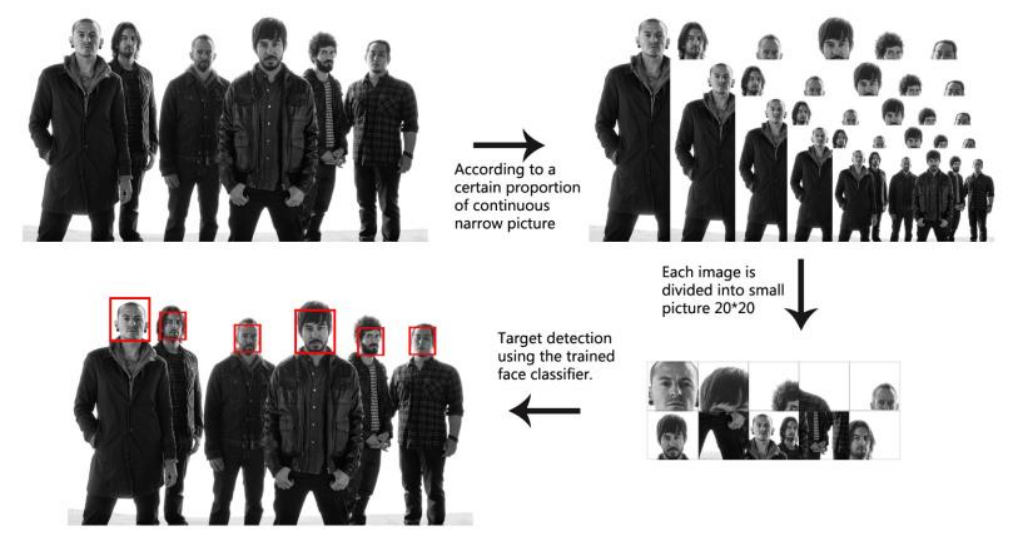

Figure 1. The Flow Chart of OpenCV Face Detection

\section{Mobile Augmented Reality}

\subsection{Development}

Augmented Reality, AR for short, is a kind of new technology. It uses computer system to produce the 3D information to enhance the user to perceive the physical world. Entity information (visual, sound, smell, touch, etc.,) which is difficult to experience originally in the real world within the scope of a certain time and space, can be perceived by human senses through the simulation of science and technology after stack to the real world, so that the human sensory experience get beyond reality. AR technology can be traced back to the earliest Boeing auxiliary wiring system design. Special-helmeted engineers can see wiring path and writing tip, superimposed on the actual field. So this greatly reduced the complexity of the disassembly. Although the augmented reality hasn't popularization and development of the scale, but with effective integration of technology, content and application scenarios, and the development of hardware performance and the rise of wearable 
devices, AR has been gradually applied to every aspect of daily life, whose future development space is great.

In recent years smartphones became extremely popular, it changed the appearance of AR. Equipped with a combination of computing power, video, video display, and additional functions such as GPS, network, touch, and the price of the civilian, so researches on using smartphones as a platform of AR have becoming more and more. Recently, dozens of developers of mobile augmented reality application expect AR technology as a next big thing of mobile market. A report, recently published by Juniper Research, predicts that mobile AR market annual sales revenue will increase from less than \$1 million in 2009 to $\$ 732$ million in 2014. On December 3, 2014, Microsoft officially publicly the patent called Mobile Platform for Augmented Reality. Thus, mobile augmented reality has become one of the hottest Web trends, whose principle diagram is shown in Figure 2.

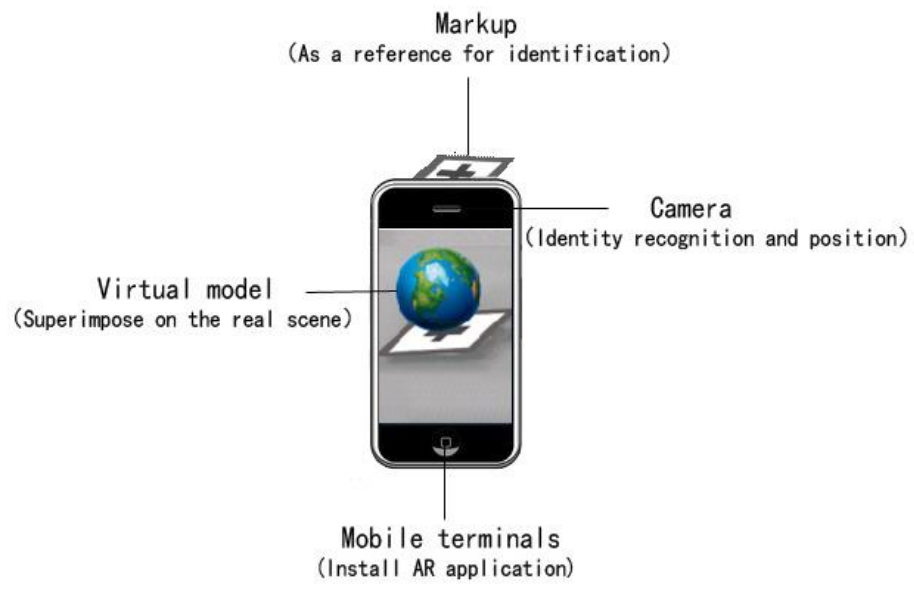

Figure 2. Principle Diagram of Mobile Augmented Reality

\subsection{Typical technology: QCAR}

QCAR (Vuforia) is a product of Qualcomm Connected Experiences; Inc. QCAR is the software platform that enables the best and most creative branded augmented reality app experiences across the most real world environments, giving mobile apps the power to see. QCAR uses superior, stable, and technically efficient computer vision-based image recognition and offers the widest set of features and capabilities, giving developers the freedom to extend their visions without technical limitations. With support for IOS, Android, and Unity 3D, QCAR allows you to write a single native app that can reach the most users across the widest range of smartphones and tablets. With interactive design and game development experience as long as the download Vuforia SDK for developers, using standard writing process computer components to drag and drop to the Unity of development can be achieved.

Smart Terrain technology of QCAR for smartphones and tablet PCS is a major breakthrough in the field of augmented reality experience. Based on Smart Terrain technology applications to make the virtual characters have the ability to interact with the physical world in an unprecedented way, it brings brand-new interaction level and realistic experience to users. McDonald's is the first global brand of using the Smart Terrain technology in commercial application. Developed jointly by the Trigger and DDB Chicago McDonald's GOL! Mobile application is an all-new QCAR based virtual stunt football game, allow players to experience digital games in real environment. At present, the award-winning QCAR has become a hot technology that has surprised the world. QCAR global ecological system has more than 100,000 registered from 130 countries developers. There are more than 9000 
IOS and Android terminal application based on QCAR in different domains such as stores, consumer goods, activities and mobile phone games.

The purpose of this study is how to face detection technology and the organic combination of mobile augmented reality. On the basis of QCAR and OpenC, Va development framework of the face detection technique based on OpenCV in mobile augmented reality application is putting forward. Using OpenCV to compensate for QCAR weaknesses in image processing, and AR face book as an example(execution flow chart shown in Figure 3), analyzes the key technology in the process of development based on face detection in mobile augmented reality application.

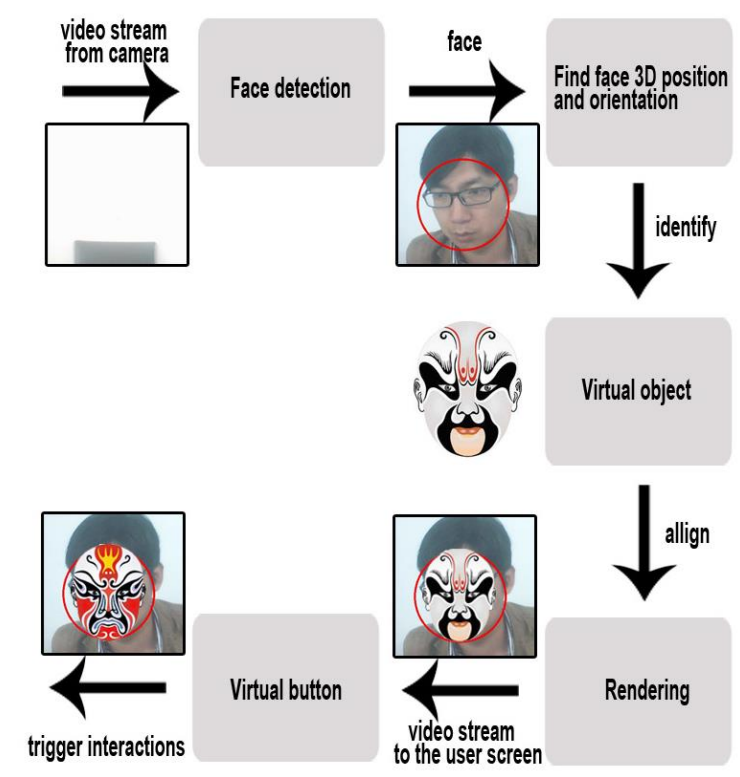

Figure3. Execution Flow Chart of AR Face Book

\section{Development Framework}

According to AR face book execution flow chart analysis, the AR interactions in mainly involves four aspects of application development, including face detection (VS2010+OpenCV), 3d model (3ds MAX), AR interaction design (Unity 3D+QCAR+PlayMaker), Android applications (SDK). According to the personal development experience, with Unity $3 \mathrm{~d}$ as the middleware of the organic combination of four types of application development, in this paper, a development framework of augmented reality interactive system on face detection is based on Android platform is proposed. This development framework consists of five modules: overall design, model making, face detection, AR design, testing, as shown in Figure 4. 


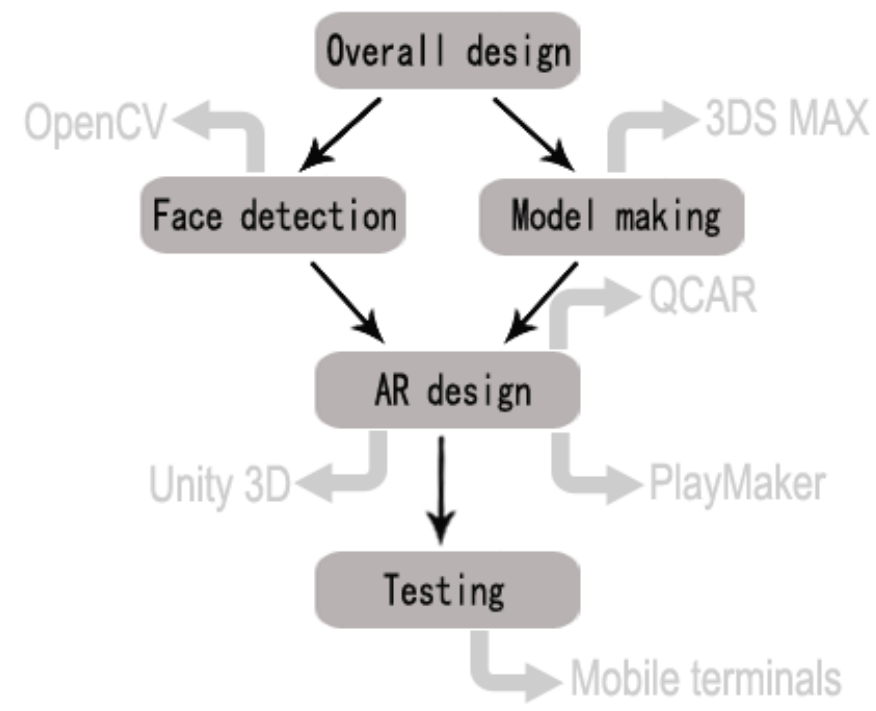

Figure 4. Development Framework

\section{Key Parsers-- AR Face Book, for Example}

\subsection{Overall Design}

This module proposes the overall design scheme of AR face book, which is shown $3 \mathrm{~d}$ face on the face position in the camera, and use touch for human-computer interaction to achieve a similar effect of Sichuan Opera face.

\subsection{Face Detection}

Function of face detection procedure is completed in three parts, namely: loading classifier; loading the image to be detected; detection and labeling. This program uses the target detection classifier, which is stored in " haarcascade_frontalface_alt.xml" file provided by OpenCV. After the classifier loaded by cvLoad function and casts. cvHaarDetectObjects is the function, which is used to detect the image targets in OpenCV. Function uses training cascade classifier of target object (such as face) to detect rectangular area including target objects in the image, and return these regions as a sequence of rectangle. Classifier is explicitly release by cvReleaseHaarClassifierCascade funtion after use. In VS2010, build project, edit the code, compiled after the operation.Faces in the camera can be detected and marked by red circle, as shown in Figure 5. The key code is as follows:

Faces $=$ cvHaarDetectObjects (small_img, FDcascade, FDstorage,

1.1 ,

3 ,

0 ,

//CV_HAAR_DO_CANNY_PRUNING

//CV_HAAR_FIND_BIGGEST_OBJECT

CV_HAAR_DO_ROUGH_SEARCH

CV_HAAR_SCALE_IMAGE

0 ,

cvSize $(20,20))$; 


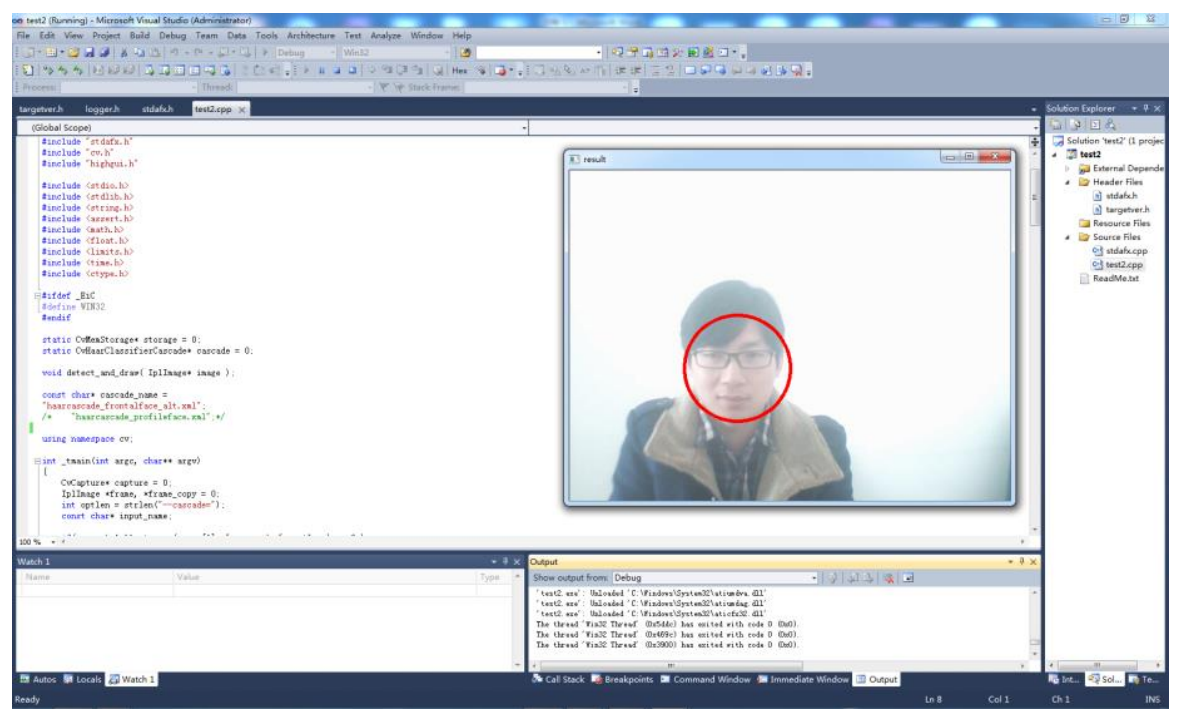

Figure 5. OpenCV Face Detection

\subsection{Model Making}

According to the overall design scheme and material collection, image processing of Photoshop can be used as the map of a 3D model. Make 3D model of face book., set up texture, make animation, rendering, by using 3ds MAX as shown in Figure 6. Due to the particularity of mobile devices, so as far as possible with minimal surface modeling of the best effect, otherwise may lead to cell phone run-time speed can be slow or even collapse. So when modeling is a good optimization of the model, so that users can obtain the realtime $3 \mathrm{~d}$ experience.

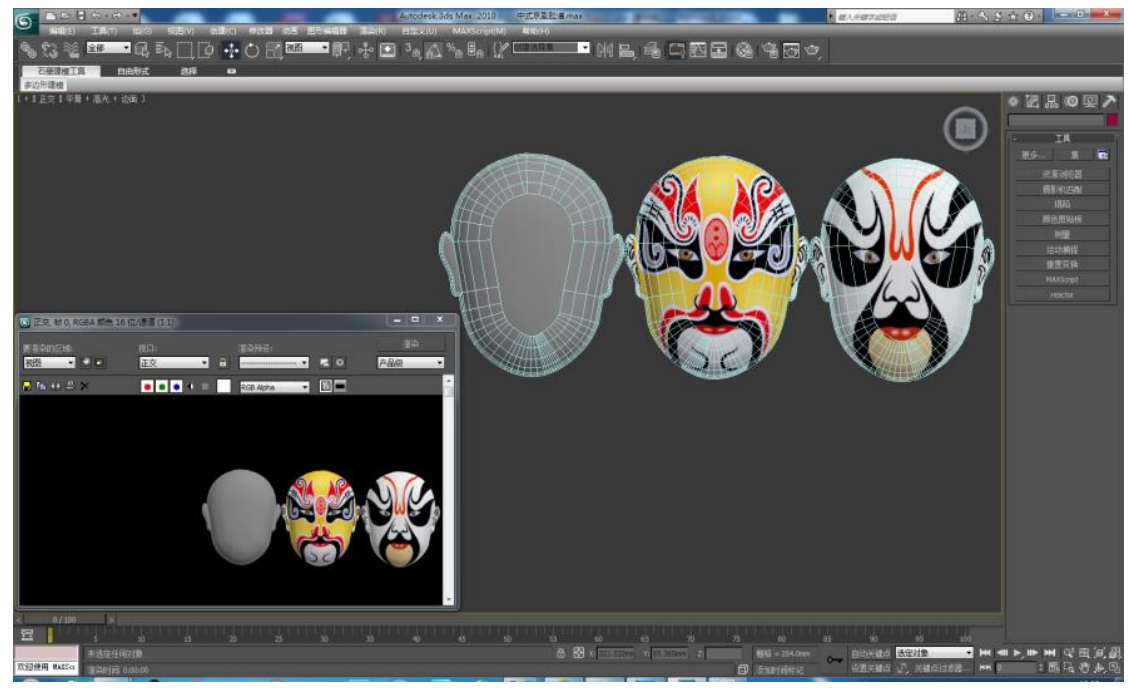

Figure 6. 3d Modeling and Rendering

\subsection{AR Design}

AR design is the most important part of the program. According to the overall design, the research will first compiled OpenCV face detection program used in Unity $3 \mathrm{~d}$, in order to realize the face detection; then reuse QCAR plug-in for augmented reality design, add $3 \mathrm{~d}$ faces and virtual buttons and the corresponding position; finally use the Playmaker plug-in for the virtual button to set the interaction, realize $3 \mathrm{~d}$ face model material replacement, as shown Figure 7. Sichuan opera face effect is achieved. 


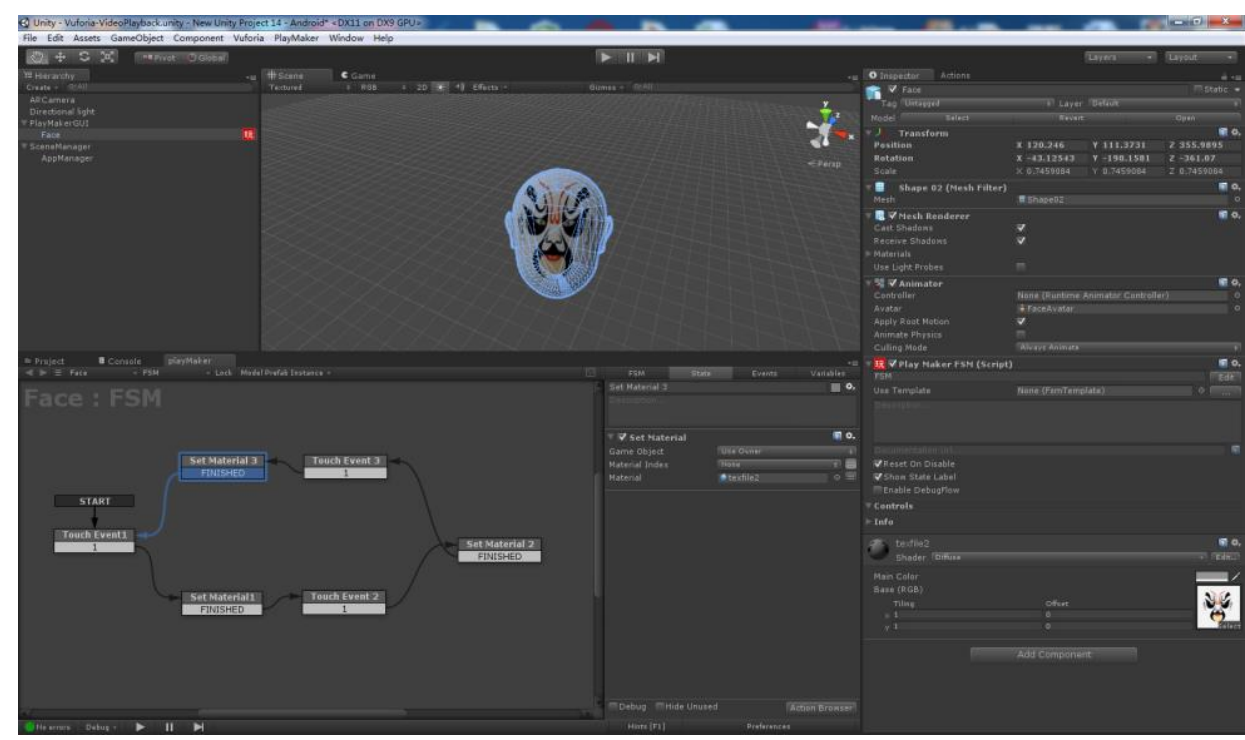

Figure7. AR Interactive Design

\subsection{Testing}

After installing the APK released by unity $3 \mathrm{~d}$ in the Android mobile phone, aim the camera to face into testing. If detect the human face, $3 \mathrm{~d}$ faces will be displayed in the corresponding position, and can move along with the movement of face. By touching the screen, users can interact with the $3 \mathrm{~d}$ face; realize the effect of Sichuan opera face. According to the visualization and interactive real-time to optimize the $3 \mathrm{~d}$ model setup, including model, texturing and lighting, etc., until you can program on the android mobile phone testing and interacting smoothly, as shown in Figure 8.
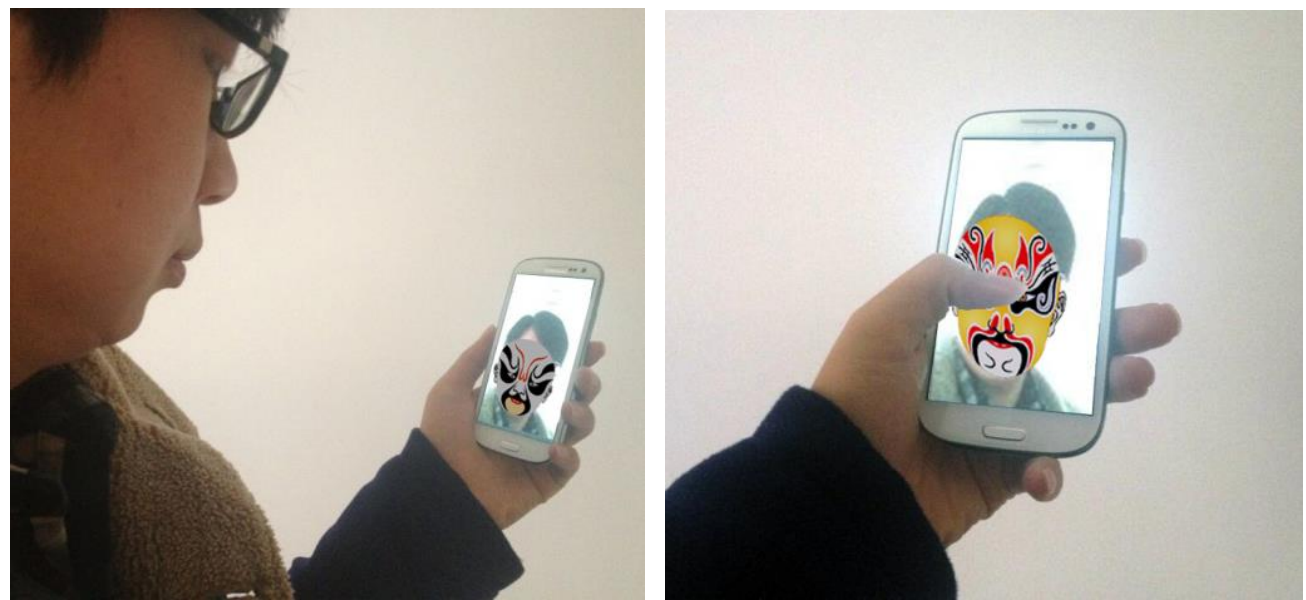

Figure 8. Operation Effect of AR Face Book

\section{Summary}

In this paper, combining face detection technology and mobile augmented reality is applied to research. And with OpenCV and QCAR as typical technology, AR face book is developed on the android platform which is an interactive programs of mobile augmented reality based on face detection. And implementation of the key parts in the process of application development is expounded. Although the development of the intelligent mobile phone and AR technology has simple face 
detection can be realized in mobile AR application, it still faces many technical challenges compared with the PC work platform, such as low image resolution and graphics processing power, limited screen size and storage space, etc., which have become obstacles of popularization and realization of the technology. Therefore, the performance of smartphone is unable to compete with a PC in the up-coming future. The lagging behind of smartphone on hardware development makes the algorithm running on a PC cannot be effective transplantation, drives a further wedge between mobile AR technologies with traditional AR technology in the development speed of gap.

However, with the continuous development and improvement of mobile terminal technology, the constraints of restricting AR technology transplanted to the smartphone platform will become the past. Smartphones as part of the people's daily life will play a greater technical advantage and function. And the combination of face detection technology, AR technology and mobile terminals will open a new research direction in the field of AR application.

\title{
References
}

[1] S. Li, Y. Liu and X. Zhiyong, "Face recognition principle and algorithm", Posts \& Telecom Press, Beijing, china, (2014).

[2] R. Laganiere, "OpenCV2 Computer Vision Application Programming Cookbook", Science Press, Beijing, china, (2013).

[3] Z. Ying, L. Yong-Ping and A. O. Xin-Yu, "Design of a Common Face Detection Module Based on OpenCV", Computer Engineering \& Science, vol. 10, (2011)

[4] L. Liang, Y. Ke, W. Yong-Tian, L. Yue, "Key Issues Study for Mobile Augmented Reality System", Journal of Image and Graphics, vol. 3, (2009).

[5] C. Yongm, X. Limingm and J. Shufang, "Research on key technology of smart phone augmented reality system", Computer Engineering and Applications, vol. 47, (2011).

[6] M. Menard, "Game Development with Unity", Course Technology PTR, Boston, USA, (2011).

[7] G. Lei and W. Qiu-Guang, "Research of face detection based on Adaboost algorithm and openCV implementation", Journal of Harbin University of science and Technology, vol. 14, (2009).

[8] C. Jing, W. Yong-Tian, G. Jun-Wei and L. Wei, “Augmented Reality Technology Applied on Mobile Phone Platform", Journal of University of Electronic Science and Technology of China, vol. 4, (2010).

\begin{abstract}
Authors
Hu Peng, He received his M.Sc. in Computer software and theory (2008) from Kunming University of Science and Technology. Now he is full lecturer at Oxbridge College, Kunming University of Science and Technology. His current research interests include different aspects of virtual reality technology.
\end{abstract}

